\title{
Charming loops in exclusive rare FCNC $B$-decays
}

\author{
Dmitri Melikhov ${ }^{1,2,3, *}$ \\ ${ }^{1}$ D. V. Skobeltsyn Institute of Nuclear Physics, M. V. Lomonosov Moscow State University, 119991, \\ Moscow, Russia \\ ${ }^{2}$ Institute for High Energy Physics, Austrian Academy of Sciences, Nikolsdorfergasse 18, A-1050 Vi- \\ enna, Austria \\ ${ }^{3}$ Faculty of Physics, University of Vienna, Boltzmanngasse 5, A-1090 Vienna, Austria
}

\begin{abstract}
Rare $B$-decays induced by flavour-changing neutral currents (FCNC) is one of the promising candidates for probing physics beyond the Standard model. However, for identifying potential new physics from the data, reliable control over QCD contributions is necessary. We focus on one of such QCD contributions - the charming loops - that potentially can lead to difficulties in disentangling new physics effects from the observable and discuss the possibility to gain control over theoretical predictions for charming loops.
\end{abstract}

\section{Introduction}

The interest in the contributions of charm to rare FCNC decays of the $B$-mesons is to a great extent motivated by the fact that virtual charm-quark loops, including charmonia states which appear in the physical region of several FCNC $B$-decay, have a strong impact on the $B$-decay observables [1] thus providing a "noise" for the extraction of possible new physics effects. FCNC decays are forbidden in the SM and proceed through loop contributions, see Figure 1.

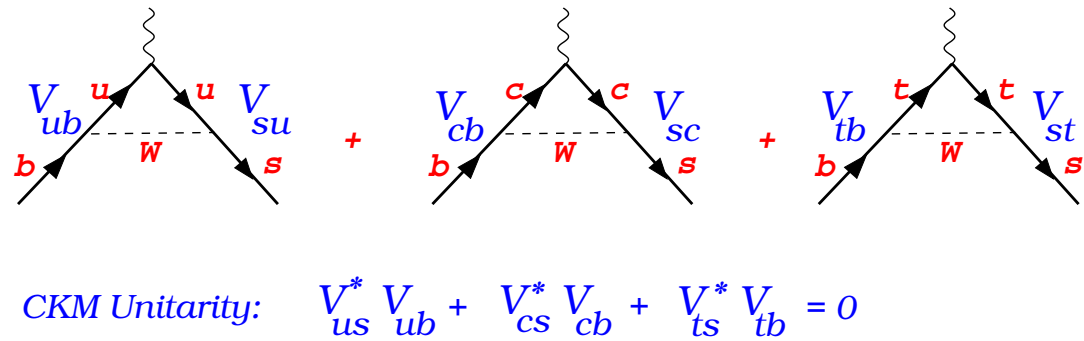

Figure 1. The amplitude of a rare FCNC $b \rightarrow s \gamma$ transition in the SM. The process proceeds via the contribution of positive-charged quarks in the loops. Recall that the unitarity of the CKM matrix leads to the cancellation of the leading ultraviolet divergences in the sum of the penguin diagrams.

The $u$-quark contribution to the CKM unitarity is strongly suppressed, leading to

$$
V_{t b} V_{t s}^{*} \simeq-V_{c b} V_{c s}^{*}
$$

*e-mail: dmitri_melikhov@gmx.de 
so the contributions of the top and the charm have approximately the same CKM strength, while the $u$-quark contribution in the loop may be neglected.

\subsection{Top-quark contribution to FCNF $B$-decays}

In $B$-decays, the characteristic energy scale $\mu \sim m_{b}$ is much lower than the masses of the $t$-quark and the $W$ and $Z$ bosons, therefore these heavy degrees of freedom may be integrated out [2-5]. As the result, e.g. for the top contribution to the effective Hamiltonian describing

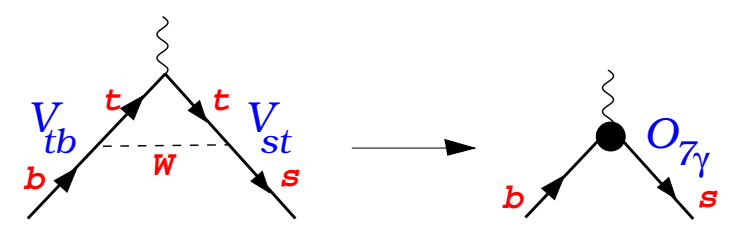

Figure 2. The $t$-quark electromagnetic penguin in the amplitude of a rare FCNC $b \rightarrow s \gamma$ transition in the SM. Integrating out the $t$-quark and $W$-boson contributions lead to the local operator $O_{7 \gamma}=$ $\bar{s} \sigma_{\mu \nu}\left(1+\gamma_{5}\right) b \cdot F^{\mu \nu}$.

the $b \rightarrow s \gamma$ decay, Fig. 2, one finds the following expression:

$$
H_{\mathrm{eff}}^{b \rightarrow s \gamma}(\mathrm{top})=-\frac{G_{F}}{\sqrt{2}} V_{t b} V_{t s}^{*} C_{7 \gamma}(\mu) \frac{e}{8 \pi^{2}} m_{b} \cdot \bar{s} \sigma_{\mu \nu}\left(1+\gamma_{5}\right) b \cdot F^{\mu v}
$$

To calculate the effective Hamiltonian for the $b \rightarrow s l^{+} l^{-}$transition, one takes into account the contribution generated by the electromagnetic penguin operator (2), and adds the contribution of box and penguin diagrams described by operators $O_{9 V}$ and $O_{10 A}$ (Fig. 3) yielding

$$
\begin{aligned}
& H_{\mathrm{eff}}^{b \rightarrow s l^{+} l^{-}} \text {(top) }=\frac{G_{F}}{\sqrt{2}} \frac{\alpha_{\mathrm{em}}}{2 \pi} V_{t b} V_{t s}^{*}\left[-2 i m_{b} \frac{C_{7 \gamma}(\mu)}{q^{2}} \cdot \bar{s} \sigma_{\mu \nu} q^{\nu}\left(1+\gamma_{5}\right) b \cdot \bar{l} \gamma^{\mu} l\right. \\
& \left.+C_{9 V}(\mu) \cdot \bar{s} \gamma_{\mu}\left(1-\gamma_{5}\right) b \cdot \bar{l} \gamma^{\mu} l+C_{10 A}(\mu) \cdot \bar{s} \gamma_{\mu}\left(1-\gamma_{5}\right) b \cdot \bar{l} \gamma^{\mu} \gamma_{5} l\right] .
\end{aligned}
$$

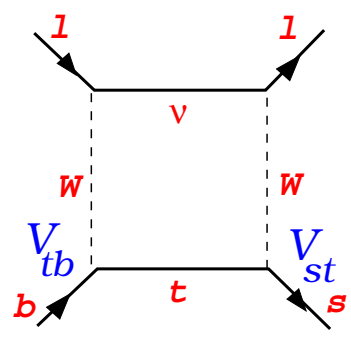

(a)

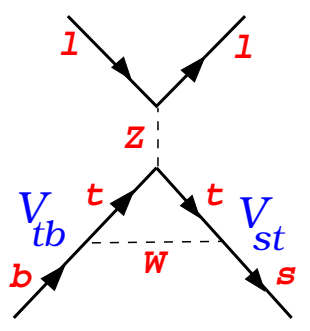

(b)

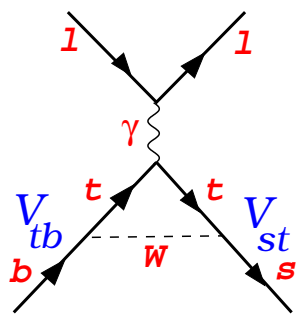

(c)

Figure 3. The amplitude of the rare FCNC $b \rightarrow s l^{+} l^{-}$transition in the SM. After integrating our $t$, $W$, and $Z$, diagrams (a) and (b) are reduced to local operators $O_{9 V}$ and $O_{10 A}$, whereas diagram (c) is proportional to $O_{7 \gamma}$. 


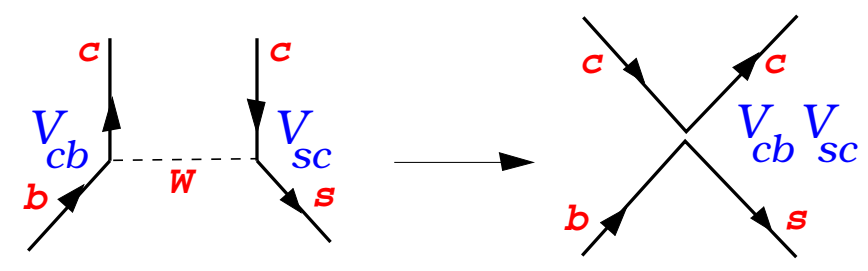

Figure 4. The amplitude of the rare FCNC $b \rightarrow s l^{+} l^{-}$transition in the SM. After integrating our $t$, $W$, and $Z$, diagrams (a) and (b) are reduced to local operators $O_{9 V}$ and $O_{10 A}$, whereas diagram (c) is proportional to $O_{7 \gamma}$.

\subsection{Charm-quark contribution to FCNC $B$-decays}

The $c$-quark is dynamical at the scale $\mu \simeq m_{b}$, so at most one can integrate out the $W$-boson contribution and come to the four-fermion effective Hamiltonian

$$
H_{\mathrm{eff}}^{b \rightarrow s \bar{c} c}=-\frac{G_{F}}{\sqrt{2}} V_{c b} V_{c s}^{*}\left\{C_{1}(\mu) O_{1}+C_{2}(\mu) O_{2}\right\}
$$

with

$$
O_{1}=\bar{s}^{j} \gamma_{\mu}\left(1-\gamma_{5}\right) c^{i} \bar{c}^{i} \gamma^{\mu}\left(1-\gamma_{5}\right) b^{j}, \quad O_{2}=\bar{s}^{i} \gamma_{\mu}\left(1-\gamma_{5}\right) c^{i} \bar{c}^{j} \gamma^{\mu}\left(1-\gamma_{5}\right) b^{j},
$$

The SM Wilson coefficients at the scale $\mu_{0}=5 \mathrm{GeV}$ have the values [corresponding to $\left.C_{2}\left(M_{W}\right)=-1\right][4,5]: C_{1}\left(\mu_{0}\right)=0.241, C_{2}\left(\mu_{0}\right)=-1.1, C_{7}\left(\mu_{0}\right)=0.312, C_{9 V}\left(\mu_{0}\right)=-4.21$, $C_{10 A}\left(\mu_{0}\right)=4.41$.

\subsubsection{Factorizable charm contributions}

Using Eq. (4), factorizable charm contributions to the $b \rightarrow$ sll amplitude reads [13]:

$$
\begin{aligned}
H_{\mathrm{eff}}^{b \rightarrow s l^{l^{+} l^{-}}} \text {(charm, fact) }=\frac{G_{F}}{\sqrt{2}} & \frac{\alpha_{\mathrm{em}}}{2 \pi} V_{c b} V_{c s}^{*}\left(C_{2}+3 C_{1}\right) g_{c c}\left(\hat{m}_{c}^{2}, \hat{q}^{2}\right) \\
& \times\left(\bar{s} \gamma_{\mu}\left(1-\gamma_{5}\right) b\right)\left(\bar{l} \gamma^{\mu} l\right),
\end{aligned}
$$

where $\left.g_{c c}\left(\hat{m}_{c}^{2}, \hat{q}^{2}\right), \hat{m}_{c}=m_{c} / m_{b}, \hat{q}^{2}=q^{2} / m_{b}^{2}\right)$, is the function describing charm contribution to vacuum polarization. At leading order in $\alpha_{s}$, one has

$$
g_{c c}\left(\hat{m}_{c}^{2}, 0\right)=-\frac{8}{9} \log \left(\frac{m_{c}}{m_{b}}\right)-\frac{4}{9} \sim 1 \text {. }
$$

The factorizable charm contribution to the $b \rightarrow s l^{+} l^{-} l$ effective Hamiltonian has the same Lorentz structure as the $C_{9 V}$ term in the top contribution, Eq. (3). So, the relative strength of the factorizable charm/top contributions is essentially determined by the ratio of the Wilson coefficients $C_{2}+3 C_{1}$ and $C_{9 V}$ (obvioulsly, the top contribution contains also other Lorentz structures, therefore the total top contribution is further enhanced; also the charm contribution contains also nonfactorizable pieces to be discussed later; these are proportional to $C_{2}$ ). The combination $C_{2}+3 C_{1}$ depends strongly on the precise value of the low-energy scale $\mu \sim m_{b}$. For instance, at $\mu=m_{b}$, one finds $C_{2}+3 C_{1}=-0.3$, while $C_{9 V}=-4.21$. Indeed, there is some numerical (although not parametric) suppression of the factorizable charm contribution but this suppression is the subject to the precise choice of the scale $\mu$. This indicates the importance of higher-order QCD corrections. 
As the final step, the calculation of the amplitude of an exclusive FCNC $B$-decay requires the $B$-meson weak decay form factors. For instance, for $B \rightarrow \gamma l^{+} l^{-}$decay, one needs the $B \rightarrow \gamma$ transition form factors

$$
\begin{aligned}
\left\langle\gamma^{*}(k, \epsilon)\left|\bar{s} \gamma_{\mu} \gamma_{5} b\right| \bar{B}_{s}(p)\right\rangle & =i e \epsilon_{\alpha}^{*}\left(g_{\mu \alpha} k^{\prime} k-k_{\alpha}^{\prime} k_{\mu}\right) \frac{F_{A}\left(k^{\prime 2}, k^{2}\right)}{M_{B_{s}}}, \\
\left\langle\gamma^{*}(k, \epsilon)\left|\bar{s} \gamma_{\mu} b\right| \bar{B}_{s}(p)\right\rangle & =e \epsilon_{\alpha}^{*} \epsilon_{\mu \alpha k^{\prime} k} \frac{F_{V}\left(k^{\prime 2}, k^{2}\right)}{M_{B_{s}}}, \\
\left\langle\gamma^{*}(k, \epsilon)\left|\bar{s} \sigma_{\mu \nu} \gamma_{5} b\right| \bar{B}_{s}(p)\right\rangle k^{\prime \nu} & =e \epsilon_{\alpha}^{*}\left(g_{\mu \alpha} k^{\prime} k-k_{\alpha}^{\prime} k_{\mu}\right) F_{T A}\left(k^{\prime 2}, k^{2}\right), \\
\left\langle\gamma^{*}(k, \epsilon)\left|\bar{s} \sigma_{\mu \nu} b\right| \bar{B}_{s}(p)\right\rangle k^{\prime \nu} & =i e \epsilon_{\alpha}^{*} \epsilon_{\mu \alpha k^{\prime} k} F_{T V}\left(k^{\prime 2}, k^{2}\right) .
\end{aligned}
$$

We have already noticed that the strong sensitivity of the combination $\left(3 C_{1}+C_{2}\right)$ to the precise value of the scale $\mu$ indicates the importance of higher-order QCD corrections. The latter should be synchronously taken into account in the Wilson coefficients and in the $B$ decay form factors; this is however a very subtle and difficult problem.

\subsubsection{Nonfactorizable charm contribution}

In addition to factorizable charm contributions, one needs to take into account nonfactorizable charm effects induced by soft gluons emitted from the charm-quark loop and absorbed in the $B$-meson loop. The latter are not reduced to the product of charm contribution to the vacuum polarization and the meson transition form factors, but have a more complicated structure. Nonfactorizable charm contributions to the effective Hamiltonian $H_{\text {eff }}^{b \rightarrow l^{+} l^{-}}$should be obtained as the convolution of the octet-octet part of the effective $b \rightarrow s \bar{c} c$ effective Hamiltonian

$$
H_{\mathrm{eff}}^{b \rightarrow s \bar{c} c}(\text { octet }- \text { octet })=\frac{G_{F}}{\sqrt{2}} V_{c b} V_{c s}^{*} \frac{C_{2}}{6}\left(\bar{s} \gamma_{\mu}\left(1-\gamma_{5}\right) T^{L} b\right)\left(\bar{c} \gamma_{\mu}\left(1-\gamma_{5}\right) T^{L} c\right),
$$

with the electromagnetic

$$
i e Q_{c}\left(\bar{c} \gamma_{\alpha} c\right) A_{\alpha}^{e . m .}
$$

and the strong

$$
i g_{s}\left(\bar{c} \gamma_{\beta} T^{N} c\right) A_{\beta}^{N}
$$

vertices of the charm-quark field. Here $A_{\alpha}^{e . m .}$ and $A_{\beta}^{N}$ are the photon and the gluon field, respectively; $T^{L}=\lambda^{L} / 2$, with $\lambda^{L}$ the Gell-Mann matrices, the indices $L, N=1, \ldots, 8$.

Nonfactorizable charm contributions are governed by a large Wilson coefficient $C_{2}$. So, nonfactorizabe and factorizable charm-loop effects are expected to be of the same order of magnitude. Comparing the patterns of broad charmonia measured in $l^{+} l^{-}$collisions and in $B \rightarrow\left(K, K^{*}\right) l^{+} l^{-}$decays, one concludes that in the charmonia region, nonfactorizable gluon exchanges are indeed at least equivalently important as factorizable charm effects.

A number of theoretical analyses of nonfactorizable effects induced by charm-quark contributions has been published in the literature: an effective gluon-photon local operator describing the charm-quark loop has been calculated in [6] for the real photon as an expansion in inverse charm-quark mass $m_{c}$ and applied to inclusive $B \rightarrow X_{s} \gamma$ decays; Ref. [7] obtained a nonlocal effective gluon-photon operator for the virtual photon and applied it to inclusive $B \rightarrow X_{s} l^{+} l^{-}$decays. In [8] nonfactorizable corrections in exclusive FCNC $B \rightarrow K^{*} \gamma$ decays using local OPE have been studied; in $[9,10]$, these corrections have been analyzed with light-cone sum rules using local OPE for the photon-gluon operator and three-particle light-cone distribution amplitudes of $K^{*}$-meson. 
As emphasized in $[6,7,11-13]$, local OPE for the charm-quark loop leads to a power series in $\Lambda_{\mathrm{QCD}} m_{b} / m_{c}^{2}$ (see Appendix A). This parameter is of order unity for the physical masses of $c$ - and $b$-quarks and thus corrections of this type require resummation. The authors of [13] derived a different form of the nonlocal photon-gluon operator compared to [7] and evaluated its effect at small values of $q^{2}$ ( $q$ momentum of the lepton pair) making use of light-cone 3-particle DA (3DA) of the $B$-meson with the aligned arguments, $\left\langle 0\left|\bar{s}(y) G_{\mu \nu}(u y) b(0)\right| B_{s}(p)\right\rangle$. In [14] it was shown that the consistent resummation of $\left(\Lambda_{\mathrm{QCD}} m_{b} / m_{c}^{2}\right)^{n}$ terms requires the generic 3DA of the $B$-meson, $\left\langle 0\left|\bar{s}(y) G_{\mu \nu}(x) b(0)\right| B_{s}(p)\right\rangle$, with non-aligned coordinates.

In the next sections we discuss in detail the contributions of charm to the amplitudes of excusive FCNC $B$-decays. To avoid technical complication related to a Lorentz/spinorial structure of the amplitudes, we illustrate the calcuation using a field theory with scalar quarks/gluons; the generalization to QCD is straightforward. We nevertheless keep the QCD notations for quark fields and assume the following hierarchy of quark masses $m_{b} \gg m_{c} \gg$ $m_{s} \sim \Lambda_{\mathrm{QCD}}$, and adopt the counting scheme in which the parameter $\Lambda_{\mathrm{QCD}} m_{b} / m_{c}^{2}$ is kept of order unity.

\section{Factorizable charm-loop effects}

Factorizable charm-loop contributions are reduced to the product of the $B$-meson weak transition form factors induced by bilinear $b \rightarrow s$ weak quark currents and the charm contribution to vacuum polarization, Fig. 5 . The latter quantity may be calculated in perturbation theory

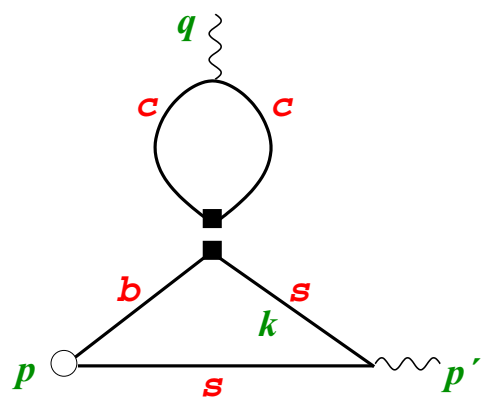

Figure 5. Factorizable contribution of the charm loop to the amplitude of an FCNC $B$-decay. The upper part of the diagram is the charm contribution to the vacuum polariization. The lower part of the diagram describes the $B$-meson decay induced by a FCNC $b \rightarrow s$ weak current. It is determined by the same form factors that describe the amplitude of an FCNC $B$-decay induced ba the top-quark contribution in the loop. The solid squares denote the color singlet-singlet four-quark operaotrs.

far below the charm quark threshold, $q^{2} \ll 4 m_{c}^{2}$. Above the charm threshold, perturbative QCD calculations are not applicable, and approaches based on hadron degrees of freedom should be used. However, vacuum polarization can be measured experimentally in a broad range of $q^{2}$, including the region above the charm threshold.

The form factor describing a FCNC transition $B_{s} \rightarrow \gamma^{*}(q) \gamma^{*}\left(p^{\prime}\right)$ is defined as follows:

$$
F\left(q^{2}, p^{\prime 2}\right)=i \int d x e^{i q x}\left\langle 0|T(\bar{s}(x) b(x), \bar{s}(0) s(0))| B_{s}(p)\right\rangle .
$$

Here $p=q+p^{\prime}$ and $p^{2}=M_{B}^{2}$, and in this formula the quark fields are Heisenberg field operators in QCD. By expanding these operators in powers of $\alpha_{s}$, one generates the corresponding expansion of the form factor. 


\subsection{Leading-order contribution}

At leading order in $\alpha_{s}$, the form factor is given by the diagram of Figure 6. This contribution to the form factor may be represented as follows:

$$
\begin{aligned}
F\left(q^{2}, p^{\prime 2}\right) & =i \int d x e^{i q x}\left\langle 0|T(\bar{s}(x) b(x), \bar{s}(0) s(0))| B_{s}(p)\right\rangle \\
& =\frac{1}{(2 \pi)^{4}} \int d x e^{i q x} d k e^{-i k x} \frac{\left\langle 0|\bar{s}(x) b(0)| B_{s}(p)\right\rangle}{m_{s}^{2}-k^{2}-i 0}
\end{aligned}
$$

Here the $B$-meson Bethe-Salpeter wave function, $\left\langle 0|\bar{s}(x) b(0)| B_{s}(p)\right\rangle$, depends on two variables $x^{2}$ and $x p$. One can parametrize it by a Fourier transform in variable $x p$ and perform the Taylor expansion in variable $x^{2}$ :

$$
\left\langle 0|\bar{s}(x) b(0)| B_{s}(p)\right\rangle=\int_{0}^{1} d \xi e^{-i p x \xi}\left\{\phi_{0}(\xi)+x^{2} \phi_{1}(\xi)+\ldots\right\}
$$

Because of the general properties of Feynman diagrams, the integration in the variable $\xi$ runs from 0 to 1 . We now insert this expansion in Eq. (12) and study the relative size of the different contributions generated by the expansion (14).

- The term proportinal to $\phi_{0}$ in (14) corresponds to $x^{2}=0$ and thus describes the light-cone configuration of the quarks inside the $B_{s}$-meson. Its contribution to the form factor (12) is easy to calculate:

$$
F\left(q^{2}, p^{\prime 2}\right)=\frac{1}{(2 \pi)^{4}} \int \frac{d x e^{i q x} \phi_{0}(\xi) d \xi e^{-i \xi p x} e^{-i k x} d k}{m_{s}^{2}-k^{2}-i 0}=\int_{0}^{1} \frac{d \xi \phi_{0}(\xi)}{m_{s}^{2}-(q-\xi p)^{2}}
$$

Taking into account that $(p-q)^{2}=p^{\prime 2}$, and thus $2 q p=p^{2}-q^{2}-p^{\prime 2}$, we obtain

$$
k^{2}=q^{2}(1-\xi)-\xi(1-\xi) M_{B}^{2}+p^{\prime 2} \xi
$$

We now have to take into account the crucial property of the light-cone two-particle distribution amplitude (2DA) $\phi_{0}(\xi)$ of the heavy meson $B_{s}$ : since the $b$-quark is heavy, it carries

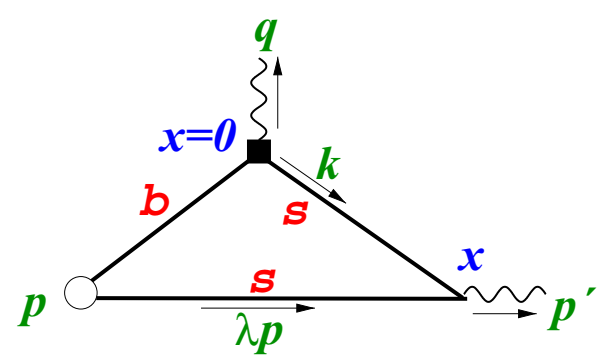

Figure 6. A schematic calculation of the $B$-decay form factor in QCD: the leading QCD contribution given in terms of the $B$-meson quark-antiquark vertex $\langle 0|b(0) q(x)| 0\rangle$. The $s$-quark line between the points $x=0$ and $y$ denotes the $s$-quark propagator, whereas the $b$-quark line and the $s$-quark line originating from the $B$-meson vertex, do not correspond to the propagators of these particles. The latter denotes the operators $b(0)$ and $\bar{s}(y)$, respectively. The light-cone configuration of the 2DA [i.e., $y^{2}=0$ ] provides the leading contribution to the form factor; deviations from the light cone lead to the contributions suppressed as $\Lambda_{\mathrm{QCD}} / m_{b}$. 
the major part of the $B$-meson momentum, such that $\phi_{0}(\xi)$ is peaked near $\xi \sim \Lambda_{\mathrm{QCD}} / m_{b}$. We then find that $k^{2} \sim-\Lambda_{\mathrm{QCD}} m_{b}$, i.e., the propagating $s$-quark is highly virtual, and therefore the perturbative expression for its propagator is well justified.

$$
F\left(q^{2}, p^{\prime 2}\right)=\int_{0}^{1} \frac{\phi_{0}(\xi) d \xi}{m_{s}^{2}-\left(q^{2}(1-\xi)-\xi(1-\xi) M_{B}^{2}+p^{2} \xi\right)}
$$

- We now turn to the $x^{2}$ terms in the expansion (14): these terms describe the deviations from the light-cone configuration. To calculate these contributions, it is convenient to substitute in Eq. [15) $x_{\alpha}=-i \frac{\partial}{\partial k_{\alpha}} e^{i k x}$. By performing the parts integration, the $k_{\alpha}$-derivative acts on the $s$-quark propagator. Taking into account that $k^{2} \sim-\Lambda_{\mathrm{QCD}} m_{b}$, we find that the contribution of a term $\left(x^{2}\right)^{n} \rightarrow\left(\Lambda_{\mathrm{QCD}} / m_{b}\right)^{n}$ compared to LC term. So the integral for the form factor (12) is indeed dominated by the light-cone quark configuration, whereas the deviations from the LC in the BS wave function of the $B$-meson are suppressed by powers of a small parameter $\Lambda_{\mathrm{QCD}} / m_{b}$.

\subsection{Next-to-leading-order contribution}

We now calculate $\alpha_{s}$-corrections generated by the expansion of the $T$-product in Eq. (12). The diagram of Fig. 7(a) corresponds to the radiative correction to the $s$-quark propagator. As we have shown above, the $s$-quark is highly virtual, so the calculation of the radiative correction is straightforward. We do not give any detail of this calculation but just notice that the corresponding contribution is again given in terms of the two-particle BS amplitude of Eq. (14). Similar to the LO contribution discussed above, the LC term in the expansion of Eq. (14) dominates the $B_{s}$-decay amplitude, whereas the terms containing powers of $\left(x^{2}\right)^{n}$ lead to power-suppressed terms $\sim\left(\Lambda_{\mathrm{QCD}} / m_{b}\right)^{n}$ in the $B_{s}$-decay amplitude.

Another correction to the $B_{s}$ decay amplitude shown in Fig. 7(b) has a different structure and needs a more complicated three-particle quark-antiquark-gluon distribution amplitude (3DA) $\left\langle 0\left|\bar{s}(y) G\left(x^{\prime}\right) b(0)\right| B_{s}(p)\right\rangle$ for its calculation. A detailed discussion of 3Da will be given

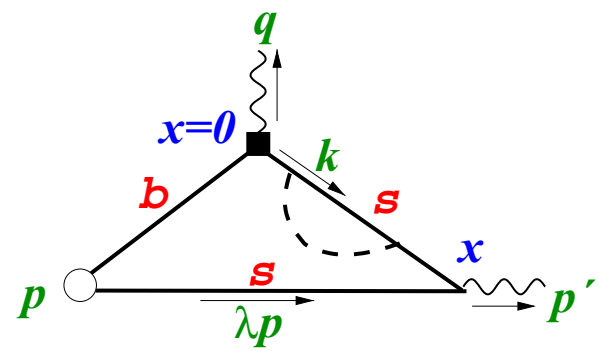

(a)

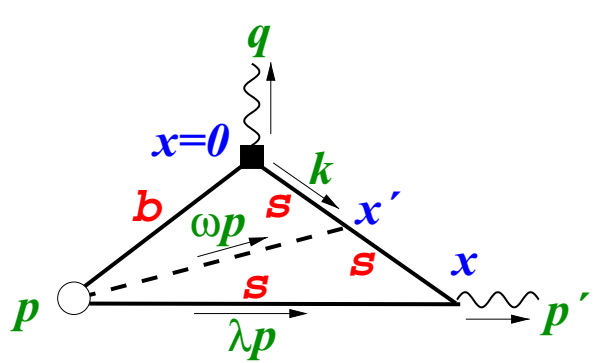

(b)

Figure 7. The NLO corrections to the $B_{s}$ weak decay form factor. (a) the NLO correction given in terms of the $B$-meson quark-antoquark vertex $\langle 0|\bar{s}(x) b(0)| 0\rangle$. (b) soft-gluon correction to the leadingorder contribution given in terms of the 3-particle DA $\langle 0| \bar{s}(x)) G\left(x^{\prime}\right) b(0)|0\rangle$. In both cases (a) and (b), the light-cone configurations of the 2DA and the 3DA [i.e., $y^{2}=0$ in the case (a) and $x^{2}=x^{\prime 2}=\left(x-x^{\prime}\right)^{2}=0$ in the case (b)] provide the leading contribution to the form factor; deviations from the light cone in both cases lead to the contributions suppressed as $\Lambda_{\mathrm{QCD}} / m_{b}$. Recall that the quark and the gluon lines attached to the $B_{s}$ meson vertex do not represent the propagators of the corresponding particls, but denote the field operators $\bar{s}(x), G\left(x^{\prime}\right)$, and $b(0)$. 
in the next Section. Here we just emphasize, that the LC term in 3DA leads to the dominant part of the $B_{s}$ decay amplitude, whereas all the terms in 3DA containing powers of $\left(x^{\prime 2}\right)^{n}$, $\left(y^{2}\right)^{n}$, and $\left(\left(x^{\prime}-y\right)^{2}\right)^{n}$, lead to the contributions to the $B_{s}$ decay amplitude, that are suppressed by powers of $\left(\Lambda_{\mathrm{QCD}} / m_{b}\right)^{n}$. So the situation is very similar to the case of the diagram of Fig. (5. This is due to the fact that the factorizable contributions discussed in this Section contain only one fermion loop containing the heavy quark $b$. We shall see in the next Section that the situation is different in the case of nonfactorizabe contributions. In the latter case, the amplitude involves two quark loops with different masses, $m_{b}$ and $m_{c}$, and this fact changes the suppression of the contributions to the $B_{s}$ amplitude, generated by the off-LC terms in the 3DA of $B_{s}$.

\section{Nonfactorizable charm-loop effects}

The charm contribution to the amplitude of an FCNC $B$-decay reads

$$
A(p, q)=i \int d z e^{i q z}\left\langle 0|T\{\bar{c}(z) c(z), \bar{s}(0) s(0)\}| B_{s}(p\rangle,\right.
$$

where quark fields are understood as Heisenberg field operators in a theory that involves weak and strong interactions. Our goal is to study nonfactorizable corrections due to a soft-gluon exchange between the charm-quark loop and the $B$-meson loop. To the lowest order, the corresponding amplitude is given by the diagram of Fig. 5:

$$
\begin{aligned}
& A(p, q)=i \int d z e^{i q z} \\
& \quad \times\left\langle 0\left|T\left\{\bar{c}(z) c(z), i \int d y^{\prime} L_{\text {weak }}\left(y^{\prime}\right), i \int d x L_{G c c}(x), \bar{s}(0) s(0)\right\}\right| B_{s}(p\rangle .\right.
\end{aligned}
$$

The effective Lagrangian that mimics weak four-quark interaction is chosen in a simple form

$$
L_{\text {weak }}=\frac{G_{F}}{\sqrt{2}} \bar{s} b \bar{c} c,
$$

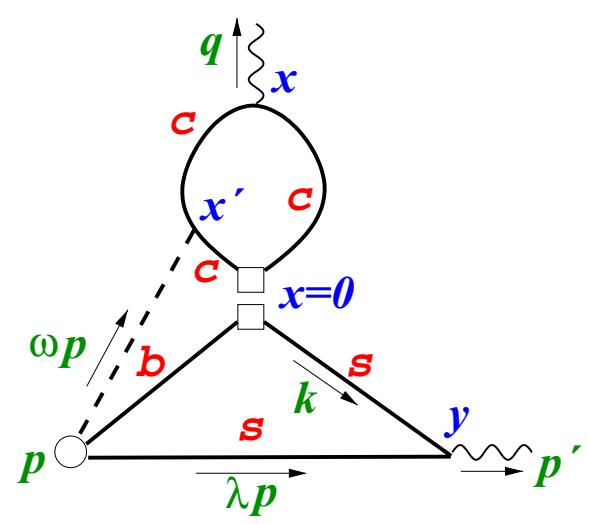

Figure 8. One of the diagrams describing the nonfactorizable gluon exchange. Dashed line corresponds to gluon; $q$ and $\kappa=-\omega p$ are the momenta outgoing from the charm-quark loop; the momentum $q^{\prime}=$ $q+\kappa=q-\omega p$ is emitted from the $b \rightarrow s$ vertex. Another diagram, equal to the one shown in the figure, corresponds to the Gluon attached to the right $c$-quark line in the upper loop. The empty squares denote the color octet-octet four-quark operators. 
while the interaction of the scalar gluon field $G(x)$ and the scalar $c$-quarks is taken to be

$$
L_{\mathrm{Gcc}}=G(x) \bar{c}(x) c(x) .
$$

\subsection{Quarks inside the charm-quark loop with soft gluon emission are perturbative}

The charm contribution is described by the three-point function. For our analysis it is important, that in the region $q^{2} \ll 4 m_{c}^{2}, q^{\prime 2}$ and $\kappa^{2}$ are also far below the charm thresholds, i.e. the charm loop is perturbative. To show that, we use the gluon field in momentum representation, which is related to the gluon field in coordinate representation as follows

$$
G(x)=\frac{1}{(2 \pi)^{4}} \int d \kappa \tilde{G}(\kappa) e^{i \kappa x}, \quad \tilde{G}(\kappa)=\int d x G(x) e^{-i \kappa x} .
$$

Then the effective operator describing the gluon emission from the charm quark loop may be written as

$$
O(q)=\int d \kappa \tilde{G}(\kappa) \Gamma_{c c}(\kappa, q)
$$

where $\Gamma_{c c}(\kappa, q)$ stands for the contribution of two triangle diagrams with the charm quark running in the loop. The momenta $\kappa$ and $q$ are outgoing from the charm-quark loop, whereas the momentum $q^{\prime}=q+\kappa$ is emitted from the $b \rightarrow s$ vertex. $p^{\prime}$ is the momentum of the outgoing $\bar{s} s$ current and $p$ is the momentum of the $B$-meson, $p=p^{\prime}+q$.

In terms of the gluon field operator in coordinate space, we can rewrite (23) as

$$
O(q)=\int d \kappa e^{-i \kappa x} d x G(x) \Gamma_{c c}(\kappa, q)
$$

\subsection{Three-particle antiquark-quark-gluon distribution amplitude of $B$-meson}

By virtue of (24), the amplitude Eq. (19) takes the form

$$
\begin{aligned}
A(q, p)=\frac{1}{(2 \pi)^{8}} \int & \frac{d k}{m_{s}^{2}-k^{2}} \int d y e^{-i\left(k-p^{\prime}\right) y} \int d x e^{-i \kappa x} \\
& \times \int d \kappa \Gamma_{c c}(\kappa, q)\left\langle 0|\bar{s}(y) G(x) b(0)| B_{s}(p)\right\rangle .
\end{aligned}
$$

Here, we encounter the $B$-meson three-particle amplitude with three (non-aligned) arguments, for which one may write down the following decomposition:

$$
\begin{aligned}
\left\langle 0|\bar{s}(y) G(x) b(0)| B_{s}(p)\right\rangle= & \int d \lambda e^{-i \lambda y p} \int d \omega e^{-i \omega x p} \\
& \times\left[\Phi(\lambda, \omega)+O\left(x^{2}, y^{2},(x-y)^{2}\right)\right] .
\end{aligned}
$$

Here $\lambda$ and $\omega$ are dimensionless variables. Making use of the properties of Feynman diagrams, one may show that they should run from 0 to 1 . However, if one of the meson constituents is heavy, it carries the major fraction of the meson momentum and as the result the function $\Phi(\lambda, \omega)$ is strongly peaked in the region

$$
\lambda, \omega=O\left(\Lambda_{\mathrm{QCD}} / m_{b}\right)
$$

So, effectively one can run the $\omega$ and $\lambda$ integrals from 0 to $\infty$; such integration limits in fact emerge in the DAs within heavy-quark effective theory $[13,15]$. We emphasize that for the results presented below only the peaking of the DAs in the region (27) is essential. Notice also that the function $\Phi(\lambda, \omega)$ in (26) coincides with the same function that appears in the "standard" 3-particle distribution amplitude with the aligned arguments, $x=u y$, discussed in [15]. 


\subsection{Light-cone contribution}

First, let us calculate the contribution to $A(q, p)$ from the term that corresponds to $x^{2}=y^{2}=$ $(x-y)^{2}=0$ in the 3DA (26). This is very easily calculable: by inserting (26) into (25) we can perform the $x$ - and $y$-integrals

$$
\begin{aligned}
& \int d x \rightarrow \delta(\kappa+\omega p), \\
& \int d y \rightarrow \delta\left(k+\lambda p-p^{\prime}\right) .
\end{aligned}
$$

Next, the $\delta$-functions above kill the integrals over $k$ and $\kappa$, and we find

$$
A(q, p)=\int_{0}^{\infty} d \lambda \int_{0}^{\infty} d \omega \Phi(\lambda, \omega) \Gamma_{c c}(-\omega p, q) \frac{1}{m_{s}^{2}-\left(\lambda p-p^{\prime}\right)^{2}} .
$$

As the result one obtains for the sum of two triangle diagrams (with the charm quark running in the loop in two opposite directions) the following expression for the amplitude:

$$
\Gamma_{c c}(\kappa, q)=\frac{1}{8 \pi^{2}} \int_{0}^{1} d u \int_{0}^{1-u} d v \frac{1}{m_{c}^{2}-2 u v \kappa q-u(1-u) \kappa^{2}-v(1-v) q^{2}} .
$$

Now, we must take into account that the $\omega$-integral is peaked at $\omega \sim \Lambda_{\mathrm{QCD}} / m_{b}$ so the gluon is soft: $\kappa=-\omega p$ and $\kappa^{2} \sim O\left(\Lambda_{\mathrm{QCD}}^{2}\right) \ll m_{c}^{2}$.

\section{4 $s$-quark produced in a weak $b \rightarrow s$ transition is highly virtual}

The momentum transferred in the weak-vertex is $q^{\prime}=q+\kappa=q-\omega p$, such that

$$
q^{\prime 2}=(q-\omega p)^{2}=q^{2}-\omega(1-\omega) M_{B}^{2}-q^{2} \omega+p^{\prime 2} \omega=q^{2}-\omega(1-\omega) M_{B}^{2} .
$$

By virtue of the $y$-integration in (28), the $s$-quark propagator takes the form

$$
m_{s}^{2}-\left(\lambda p-p^{\prime}\right)^{2}=m_{s}^{2}-\lambda q^{2}+(1-\lambda)\left(\lambda M_{B}^{2}-p^{\prime 2}\right) .
$$

Therefore, in the bulk of the $\lambda$-integration the virtuality of the $s$-quark propagator is large, $O\left(M_{B}\right)$. Therefore, the use of the Feynman expression for the $s$-quark propagator is well justified.

\subsection{Deviations from the light-cone}

We now turn to the calculation of the contributions to $A(q, p)$ generated by terms $\sim x^{2}, y^{2},(x-$ $y)^{2}$ in the 3DA (26). The terms containing powers of 4-vectors $y$ and $x$ in the integral (25) can be calculated by parts integration. Taking into account the results (28), we find the following relative contributions of the terms containing different powers of the coordinate variables:

$$
\begin{aligned}
y^{2} & \rightarrow \frac{k^{2}}{\Lambda_{\mathrm{QCD}}^{2} m_{b}^{2}} \sim \frac{1}{\Lambda_{\mathrm{QCD}} m_{b}}, \\
x^{2} & \rightarrow \frac{q \kappa}{m_{c}^{4}} \sim \frac{\Lambda_{\mathrm{QCD}} m_{b}}{m_{c}^{4}} \\
x y & \rightarrow \frac{\left(p^{\prime}-\lambda p\right)(q-\omega p)}{\Lambda_{\mathrm{QCD}} m_{b} m_{c}^{2}} \sim \frac{m_{b}}{\Lambda_{\mathrm{QCD}} m_{c}^{2}} .
\end{aligned}
$$


Clearly, all terms containing powers of $x^{2}$ and/or $y^{2}$ in the 3DA lead to the suppressed contributions to $A(q, p)$ and may be neglected within the considered accuracy. However, the terms containing powers of $x y$ lead to the contributions containing powers of $\Lambda_{\mathrm{QCD}} m_{b} / m_{c}^{2}$, i. e., of order unity within the adopted counting rules. The kinematics of the process is thus rather simple: the vectors $x$ and $y$ are directed along the light-cone [e.g., $x$ along the (+) axis, and $y$ along the (-) axis], but the 4-vector $x-y$ is obviously not directed along the light cone. Therefore, the full dependence of 3DA on the variable $(x-y)^{2}$ is needed in order to properly resum corrections of order $\left(\Lambda_{\mathrm{QCD}} m_{b} / m_{c}^{2}\right)^{n}$.

\section{Conclusions and outlook}

We reviewed the general framework for the theoretical description of semileptonic and radiative leptonic $B$-decays induced by flavour-changing neutral currents with an emphasis on gaining control over QCD effects induced by charming loops, including both factorizable and nonfactorizable contributions. The charm-loop effects are known to be small compared to the effects of the $t$-quark in the loop at small values of the dilepton pair (although there is no parametric suppression of charm compared to that of top), but dominate the amplitudes of FCNC rare $B$-decays in the region of charmonium resonances. As an illustration of the typical magnitude of the contribution of charm, we show the predicted differential distribution in $B \rightarrow l^{+} l^{-}$decays from [19] where charm is clearly seen to provide the main contribution to the branching ratios in the charmonia region. Charm contributions in the charmonia resonance region also strongly influence various observables; Fig. 9 shows the prediction for the lepton forward-backward asymmetry in $B_{s} \rightarrow \gamma l^{+} l^{-}$from [19]. The contribution of charm away from the charmonia region is expected at the level of a few percent thus providing an unpleasant "noise" for the search for the potential new physics effects. This calls for a better understanding of the charm contributions in FCNC B-decays and gaining a theoretical control over these contributions.

To put our emphasis on the conceptual aspects, we discussed the case of all scalar particles, avoiding in this way conceptually unimportant technical details.

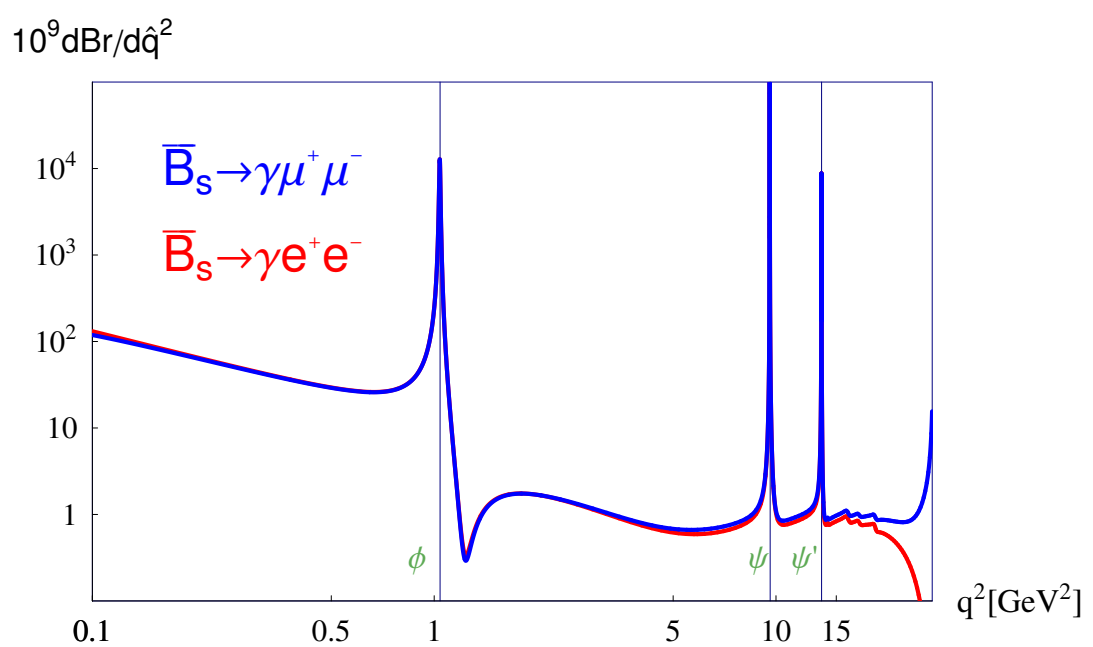

Figure 9. The predicted differential branching ratios $d \mathrm{Br} / d \hat{q}^{2}$ in $B \rightarrow \gamma l^{+} l^{-}$from [19]. $\hat{q}^{2}=q^{2} / M_{B}^{2}$. 


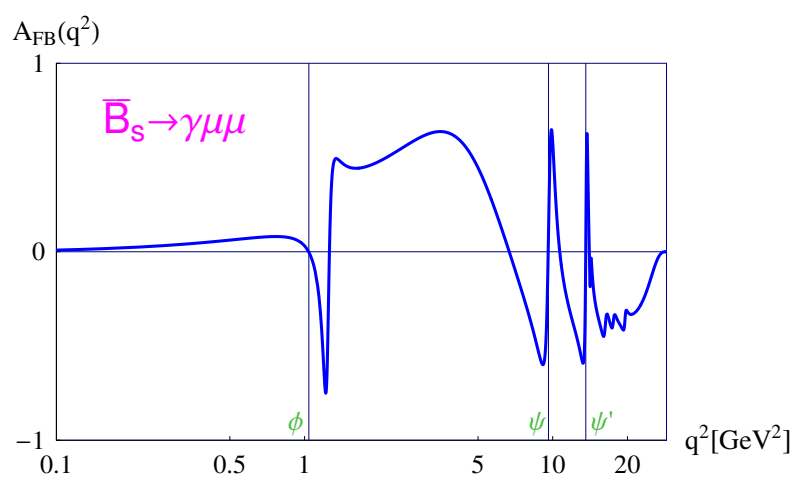

Figure 10. The predicted forward-backward asymmetry $A_{F B}\left(q^{2}\right)$ in $B \rightarrow \gamma l^{+} l^{-}$decays from [19].

Our main conclusions may be formulated as follows:

- The relevant object that arises in the calculation of the nonfactorizable corrections is the three-particle DA (or the vertex function) with independent (non-aligned) coordinates:

$$
\left\langle 0|\bar{s}(y) G(x) b(0)| B_{s}\right\rangle=\int d \lambda e^{-i \lambda y p} \int d \omega e^{-i \omega x p}\left[\Phi(\omega, \lambda)+O\left(x^{2}, y^{2},(x-y)^{2}\right)\right] .
$$

This object may be viewed as a four-point function and thus depends on five independent variables (the external momentum $p$ is on the mass shell, $p^{2}=M_{B}^{2}$ ). It is convenient to choose the variables $x p, y p, x^{2}, y^{2}$, and $(x-y)^{2}$ as these five independent variables. One may parametrize the function by its Fourier transform in the variables $x p$ and $y p$, and to write the Taylor series in the variables $x^{2}, y^{2}$, and $(x-y)^{2}$. It should be emphasized that the function $\Phi(\omega, \lambda)$ here is precisely the same function that parametrizes the standard 3DA with the aligned arguments, $x=u y$, discussed in [15].

At small $q^{2} \leq m_{c}^{2}$, terms of order $x^{2}$ and $y^{2}$ in the 3DA of $B_{s}$ meson yield small corrections to the nonfactorizable amplitude of $B$-decay compared to the LC term in the 3DA: for terms $O\left(x^{2}\right)$ the suppression parameter is $\Lambda_{\mathrm{QCD}}^{2} / m_{c}^{2}$, and for terms $O\left(y^{2}\right)$ the suppression parameter is $\Lambda_{\mathrm{QCD}} / m_{b}$. However, terms $\sim(x y)^{n}$ in the 3DA yield the contributions of order $\left(\Lambda_{\mathrm{QCD}} m_{b} / m_{c}^{2}\right)^{n}$ in the $B_{s}$ decay amplitude. These contributions are of $O(1)$ in our counting scheme and should be properly resummed. (Recall that the difference between the local OPE [6] and the light-cone OPE [13] is of the same order). We emphasize that the knowledge of merely the light-cone 3DA corresponding to $x^{2}=0, y^{2}=0$ and $(x-y)^{2}=0$, is not sufficient: While the LC 3DA allows one to resum a part of the large corrections of the order $\Lambda_{\mathrm{QCD}} m_{b} / m_{c}^{2}$, some terms of the same order remain unaccounted for. A consistent resummation of these corrections requires the knowledge of the full dependence of $\left\langle 0|\bar{s}(y) G(x) b(0)| B_{s}(p)\right\rangle$ on the variable $(x-y)^{2} .{ }^{1}$

The kinematics of the process looks simple: the 4-vectors $x$ and $y$ are directed along the light-cone [e.g., $x$ along the (+)-axis, and $y$ along the (-)-axis], but the 4-vector $x-y$ is obviously not directed along the LC; therefore, the full dependence of the 3DA (34) on the variable $(x-y)^{2}$ is needed in order to properly resum corrections of order $\left(\Lambda_{\mathrm{QCD}} m_{b} / m_{c}^{2}\right)^{n}$.

\footnotetext{
${ }^{1}$ As emphasized to us by Danny van Dyk, the actual suppression parameter of those contributions to the amplitude of FCNC $B_{s}$ decay induced by the $(x-y)^{2}$ terms in 3DA vs the LC terms in 3DA is $\Lambda_{\mathrm{QCD}} m_{b} /\left(4 m_{c}^{2}-q^{2}\right)$. For $\left|q^{2}\right| \sim m_{c}^{2}$, this agrees with our claim. If one considers the region of $q^{2} \sim-m_{b}^{2}$, then the off-LC effects turn out to be suppressed. However, whether or not for small timelike $q^{2}$ these terms may be neglected, requires further detailed analysis.
} 
- We point out that nonfactorizable soft-gluon corrections to the amplitudes of FCNC $B$ decays have qualitatively different features compared to soft-gluon corrections to the $B$ meson form factors $\langle 0|T\{\bar{s} s(z), \bar{s} b(0)\}| B(p)\rangle$, Fig. 6. In the latter case, the $B_{s}$ decay amplitudes are dominated by the light-cone configurations of quarks (and gluons) in the $B_{s}$ meson two- and three-particle vertex functions (DAs). The deviations from the LC lead to small $\sim \Lambda_{\mathrm{QCD}} / m_{b}$ corrections to the amplitudes.

- In general, when considering non-factorizable gluon corrections in meson-to-vacuum transition amplitudes of the type $\left\langle 0\left|T\left\{j_{1}(z) j_{2}(0)\right\}\right| B\right\rangle$ one encounters two distinct kinds of processes:

(i). The amplitude of the process involves only one quark loop with the valence $b$-quark. An example of this kind is the contribution to the $B_{s} \rightarrow \gamma$ form factor due to soft-gluon exchange, Fig. 6 . In this case, nonfactorizable soft-gluon correction is light-cone dominated, i.e., may be expressed via the LC 3DA of the initial $B$-meson [16-18]. The contributions to the form factor related to powers of $x^{2}, y^{2},(x-y)^{2}$ in the $B$-meson 3DA are suppressed by powers of the small parameter $\Lambda_{\mathrm{QCD}} / m_{b}$.

(ii). The amplitude of the process involves two separate quark loops (one quark-loop involving valence quarks of the initial and the final mesons and another quark loop that emits the external boson). In this case, the soft gluon from the initial heavy meson vertex is absorbed by a quark in a different loop. In this case, the description of non-factorizable soft-gluon corrections requires the full three-particle DA with non-aligned coordinates of the type of (34). Non-factorizable corrections to FCNC decays due to $c$ - or $u$-quark loops belong to this kind of processes.

\section{Acknowledgments}

Many thanks are due to Danny van Dyk for lively and interesting discussions of nonfactorizable effects in FCNC $B$-decays. I gratefully acknowledge financial support from RFBR and CNRS under joint CNRS/RFBR Grant PRC Russia/19-52-15022 and from the Austrian Science Fund (FWF), Project No. P29028. 


\section{A Local vs non-local OPE}

In this Appendix we discuss the relationship between local and non-local OPE for the amplitude of an FCNC weak decay of the $B_{s}$ meson.

To implement local OPE for the nonfactorizable part of the FCNC $B_{s}$-decay amplitude, one needs to perform the Taylor expansion of the gluon field operator $G(x)$ :

$$
G(x)=G(0)+x_{\alpha} \partial^{\alpha} G+\ldots
$$

1. The leading contribution comes from $G(0)$ term, and it can be given in terms of the 3DA with the equal coordinates of the gluon and the $b$-quark fields:

$$
\left.\langle 0|\bar{s}(y) G(0) b(0)| B(p)\rangle\right|_{x=0}=\int d \lambda e^{-i \lambda y p} \int d \omega\left[\Phi(\lambda, \omega)+O\left(y^{2}\right)\right] .
$$

2. Let us now consider the contribution of $x_{\alpha} \partial^{\alpha} G(0)$.

- The $x_{\alpha}$ factor can be generated by $-i \frac{\partial}{\partial q_{\alpha}} e^{i q x}$ and, after performing the parts integration, leads to terms proportional to $\frac{q^{\alpha}}{m_{c}^{2}}$.

- Thus, after the integration, the term containing $\partial^{\alpha} G(0)$ takes the form

$$
\begin{aligned}
\left.\frac{\partial}{\partial x_{\alpha}}\langle 0|\bar{s}(y) G(x) b(0)| B(p)\rangle\right|_{x=0} & =-i p_{\alpha} \int d \lambda e^{-i \lambda y p} \int d \omega \omega \Phi(\lambda, \omega)+C_{2} \Lambda_{\mathrm{QCD}} x_{\alpha} \\
& =C_{1} p_{\alpha} \frac{\Lambda_{\mathrm{QCD}}}{m_{b}}+C_{2} \Lambda_{\mathrm{QCD}} x_{\alpha}
\end{aligned}
$$

The term $C_{2}$ arises when the derivative acts on $x^{2}$ and $x y$ terms in the full off-LC 3DA.

3. As the next step, we must plug the derivative term (37) into the general representation for the nonfactorizable part of the $B_{s}$ amplitude (25). Now, let us compare the contributions to the amplitude (25), generated by the terms $\partial_{\alpha} G(0)$ over the $G(0)$ in the 3DA of $B_{s}$ meson. The leading part in the ratio of the $\partial_{\alpha} G(0)$ over the $G(0)$ contributions to the amplitude arises when $q_{\alpha}$ contracts with the term $\sim p_{\alpha}$ and reads

$$
\frac{q p \Lambda_{\mathrm{QCD}}}{m_{b} m_{c}^{2}} \sim \frac{M_{B} \Lambda_{\mathrm{QCD}}}{m_{c}^{2}} \sim 1
$$

For the realistic case of $c$ - and $b$-quarks, and within the adopted counting scheme $\frac{M_{B} \Lambda_{\mathrm{QCD}}}{m_{c}^{2}} \sim 1$, there is no suppression of the derivative-term contribution. So we conclude that the local OPE does not provide a hierarchy of contributions given by different operators according to their dimension. This means that a summation of infinitely many local operators is necessary in order to properly account for the terms of the type $\left(\frac{M_{B} \Lambda_{\mathrm{QCD}}}{m_{c}^{2}}\right)^{n}$. Precisely this is is done by considering the non-local OPE. 


\section{References}

[1] M. Beneke, G. Buchalla, M. Neubert, and C. T. Sachrajda, Penguins with Charm and Quark-Hadron Duality, Eur. Phys. J. C61, 439 (2009).

[2] T. Inami, and C. S. Lim, Effects of Superheavy Quarks and Leptons in Low-Energy Weak Processes $K_{L} \rightarrow \mu \bar{\mu}, K^{+} \rightarrow \pi^{+} v \bar{v}$ and $K^{0} \leftrightarrow \bar{K}^{0}$, Prog. Theor. Phys. 65, 297 (1981), Erratum: Prog. Theor. Phys. 65, 1772 (1981).

[3] B. Grinstein, M. J. Savage and M. B. Wise, $B \rightarrow X(s) e^{+} e^{-}$in the Six Quark Model, Nucl. Phys. B 319271 (1989).

[4] A. J. Buras and M. Munz, Effective Hamiltonian for $B \rightarrow X(s) e^{+} e^{-}$beyond leading logarithms in the NDR and HV schemes, Phys. Rev. D 52, 186 (1995).

[5] G. Buchalla, A. J. Buras, and M. E. Lautenbacher, Weak decays beyond leading logarithms, Rev. Mod. Phys. 68, 1125 (1996).

[6] M. B. Voloshin, Large $O\left(\mathrm{~m}_{c}^{-2}\right)$ nonperturbative correction to the inclusive rate of the decay $B \rightarrow X_{s} \gamma$, Phys. Lett. B397, 275 (1997).

[7] G. Buchalla, G. Isidori, and S. J. Rey, Corrections of order $\Lambda_{\mathrm{QCD}}^{2} / m_{c}^{2}$ to inclusive rare B decays, Nucl. Phys. B511, 594 (1998).

[8] A. Khodjamirian, R. Ruckl, G. Stoll, and D. Wyler, QCD estimate of the long distance effect in $B \rightarrow K^{*} \gamma$, Phys. Lett. B402, 167 (1997).

[9] P. Ball and R. Zwicky, Time-dependent CP Asymmetry in B $\rightarrow K^{*} \gamma$ as a (Quasi) Null Test of the Standard Model, Phys. Lett. B642, 478 (2006).

[10] J. Lyon and R. Zwicky, Resonances gone topsy turvy - the charm of QCD or new physics in $b \rightarrow s l^{+} l^{-}$? arXiv:1406.0566.

[11] Z. Ligeti, L. Randall, and M. B. Wise, Comment on nonperturbative effects in $\bar{B} \rightarrow$ $X_{s} \gamma$, Phys. Lett. B402 178 (1997).

[12] M. Benzke, S. J. Lee, M. Neubert, and G. Paz, Factorization at Subleading Power and Irreducible Uncertainties in $\bar{B} \rightarrow X_{s} \gamma$, JHEP 1008, 099 (2010).

[13] A. Khodjamirian, T. Mannel, A. Pivovarov, and Y.-M. Wang, Charm-loop effect in $B \rightarrow K^{(*)} l^{+} l^{-}$and $B \rightarrow K^{*} \gamma$, JHEP 1009, 089 (2010).

[14] A. Kozachuk and D. Melikhov, Revisiting nonfactorizable charm-loop effects in exclusive FCNC B-decays, Phys. Lett. B786, 378 (2018).

[15] H. Kawamura, J. Kodaira, C.-F. Qiao, and K. Tanaka, B-meson light cone distribution amplitudes in the heavy quark limit, Phys. Lett. B523, 111 (2001), Erratum: Phys. Lett. B536, 344 (2002).

[16] P. Ball and R. Zwicky, Improved analysis of $B \rightarrow \pi e v$ from QCD sum rules on the light cone, JHEP 10, 019 (2001).

[17] P. Ball and E. Kou, $B \rightarrow$ yev transitions from QCD sum rules on the light cone, JHEP 04, 029 (2003).

[18] A. Khodjamirian, T. Mannel, and N. Offen, B-meson distribution amplitude from the $B \rightarrow \pi$ form-factor, Phys. Lett. B620, 52 (2005).

[19] A. Kozachuk, D. Melikhov, and N. Nikitin, Rare FCNC radiative leptonic $B_{(s, d)} \rightarrow$ $\mathrm{\gamma l}^{+} l^{-}$decays in the standard model, Phys. Rev. D97, 053007 (2018). 